\title{
Counting Stirling permutations by number of pushes
}

\author{
TOUfIK MANSOUR \\ Department of Mathematics \\ University of Haifa \\ 3498838 Haifa, Israel \\ email: tmansour@univ.haifa.ac.il \\ and \\ Mark ShatTUCK \\ Department of Mathematics \\ University of Tennessee \\ Knoxville, TN 37996, USA \\ email: shattuck@math.utk.edu
}

(Received: June 10, 2019, and in revised form June 13, 2020.)

\begin{abstract}
Let $\mathcal{T}_{n}^{(k)}$ denote the set of $k$-Stirling permutations having $n$ distinct letters. Here, we consider the number of steps required (i.e., pushes) to rearrange the letters of a member of $\mathcal{T}_{n}^{(k)}$ so that they occur in non-decreasing order. We find recurrences for the joint distribution on $\mathcal{T}_{n}^{(k)}$ for the statistics recording the number of levels (i.e., occurrences of equal adjacent letters) and pushes. When $k=2$, an explicit formula for the ordinary generating function of this distribution is also found. In order to do so, we determine the $L U$-decomposition of a certain infinite matrix having polynomial entries which enables one to compute explicitly the inverse matrix.
\end{abstract}

Mathematics Subject Classification(2010). 05A15, 05A05, $15 \mathrm{~A} 06$.

Keywords: $k$-Stirling permutation, generating function, level, combinatorial statistic

\section{Introduction}

A Stirling sequence $\pi=\pi_{1} \pi_{2} \cdots$ is one that avoids the pattern 212 (i.e., there exist no indices $i<j<k$ such that $\pi_{i}=\pi_{k}>\pi_{j}$ ). Orderings of the multiset $1^{k} 2^{k} \cdots n^{k}$ where $k \geq 2$ that avoid the pattern 212 are called $k$-Stirling permutations (of order $n$ ). When $k=2$, these are the Stirling permutations originally considered by Gessel and Stanley [3]. The $k$-Stirling permutations have been provided various refined counts in the case $k=2$ (see, e.g., $[1,2,5,6]$ ) and for general $k$ (see [7]). It is seen that there are $\prod_{i=1}^{n-1}(k i+1) k$-Stirling permutations of order $n$. In this paper, we consider a new refinement of this number and in particular, when $k=2$, a new $q$-analogue of the odd double factorial sequence (see, e.g., [10, A001147]).

We now describe the combinatorial basis of our refinement. Given a word $w=w_{1} w_{2} \cdots$ on the alphabet of positive integers, we seek the number of letters that must be moved to the left so that the resulting word is non-decreasing. Consider the smallest index $i>1$ (if it exists), denoted by $i_{0}$, such that there exists at least one $j \in[i-1]$ with $w_{j}>w_{i}$. We then shift $w_{i_{0}}$ within $w$, one position at a

DOI: $10.1515 /$ puma-2015-0038

(C) 2020 Mansour, Shattuck. This is an open access article licensed under the Creative Commons Attribution-NonCommercial-NoDerivs License (http://creativecommons.org/licenses/by-nc-nd/3.0/). 
time to the left, stopping at the point when no letter occurs to the left of $w_{i_{0}}$ that is strictly greater than $w_{i_{0}}$ within the new word $w^{\prime}$. We will refer to this shifting of the letter $w_{i_{0}}$ as a push. We then repeat this procedure using $w^{\prime}$ and so on until a non-decreasing word results. By the number of pushes of a word $w=w_{1} w_{2} \cdots$, we will mean the number of steps required to achieve a non-decreasing word. Let $\mathcal{T}_{n}^{(k)}$ denote the set of $k$-Stirling permutations of order $n$. We will consider here the number of pushes as a statistic on members of $\mathcal{T}_{n}^{(k)}$, represented sequentially.

For example, if $n=5, k=3$ and $\pi=112444221355533 \in \mathcal{T}_{5}^{(3)}$, then $\pi$ has six pushes altogether corresponding to the letters 2,2,1,3 between the 4's and the 5's along with the two terminal 3's. By a weak left-right maximum (wlrm) within a sequence $s=s_{1} s_{2} \cdots s_{m}$, we mean an index $i$ such that $s_{i} \geq s_{j}$ for all $1 \leq j<i$. Note that the number of pushes of $s$ is given by $m-$ (\# wlrm) since each letter of $s$ not corresponding to a wlrm must be shifted to the left so as to achieve sorted order. Henceforth, we will consider equivalently the distribution on $\mathcal{T}_{n}^{(k)}$ for the statistic recording the number of wlrm. Note that in our example above, the permutation $\pi$ has nine wlrm altogether.

In the next section, we find recurrences satisfied by the joint distribution on $\mathcal{T}_{n}^{(k)}$ for all $k \geq 2$ for the statistics recording the number of wlrm and number of levels by appropriately decomposing the set $\mathcal{T}_{n}^{(k)}$ into disjoint parts for which a recursive structure can be found. Further recurrences are noted in the case $k=2$ corresponding to Stirling permutations. In the third section, we make use of the recurrences to compute an explicit formula for the ordinary generating function of the aforementioned joint distribution on $\mathcal{T}_{n}^{(k)}$ in the case $k=2$. Some special cases of our formula are observed.

We now briefly describe our strategy for computing the generating function in the third section. First, we introduce an infinite matrix $M$ to represent a family of recurrences satisfied by some auxiliary generating functions each involving a differential equation. We seek the inverse of $M$. While it is difficult to determine $M^{-1}$ directly, it turns out that when $M$ is expressed using the $L U$-decomposition that the individual matrices $L$ and $U$ have inverses that can be computed explicitly. Note that some guesswork is required in determining the $L U$-decomposition of $M$ and also in finding $L^{-1}$ and $U^{-1}$. Once this is done, we then have $M^{-1}=U^{-1} L^{-1}$ and are thus able to complete our derivation of the generating function formula.

\section{Recurrences for $k$-Stirling permutations}

In this section, we consider the joint distribution of the levels and weak left-right maxima statistics on $\mathcal{T}_{n}^{(k)}$. Given $\pi=\pi_{1} \pi_{2} \cdots \in \mathcal{T}_{n}^{(k)}$, we define what will be referred to as a unit of $\pi$ recursively as follows. Let $i_{1}$ be the first letter of $\pi$. Let $u_{1}$ consist of all letters of $\pi$ up to and including the last occurrence of $i_{1}$. Let $i_{2}$ (if it exists) be the letter that directly follows the last occurrence of $i_{1}$, and define $u_{2}$ similarly using the letters of $\pi$ that are not part of $u_{1}$. Successively define the units $u_{1}, u_{2}, \ldots$ until all of the letters of $\pi$ have been exhausted. For example, $\pi \in \mathcal{T}_{5}^{(3)}$ above has two units, the second starting with the first 3. Let $\mathcal{T}_{n, \ell}^{(k)}$ denote the subset of $\mathcal{T}_{n}^{(k)}$ comprising those members that contain exactly $\ell$ units for $1 \leq \ell \leq n$. See [9] for an equivalent refinement of $\mathcal{T}_{n}^{(k)}$ when $k=2$.

Let $a_{k}(n, \ell)=a_{k}(n, \ell ; p, q)$ denote the joint distribution on $\mathcal{T}_{n, \ell}^{(k)}$ for the statistics recording the 
number of levels and weak left-right maxima. That is,

$$
a_{k}(n, \ell)=\sum_{\pi \in \mathcal{T}_{n, \ell}^{(k)}} p^{\operatorname{lev}(\pi)} q^{\max (\pi)},
$$

where $\operatorname{lev}(\pi)$ is the number of levels (i.e., the number of indices $i$ such that $\left.\pi_{i}=\pi_{i+1}\right)$ and $\max (\pi)$ is the number of wlrm. The levels statistic has been previously considered on $\mathcal{T}_{n}^{(k)}$ in the case $k=2$ (see [6], where they are referred to as plateaus, and also the related paper [8]); here we consider a new bistatistic with the number of wlrm. We have the following recurrence formula for $a_{k}(n, \ell)$.

THEOREM 2.1 If $k \geq 2$ and $n \geq \ell \geq 1$, then

$$
\begin{aligned}
a_{k}(n, \ell)= & p^{k-1}\left(\ell-1+q^{k}\right) a_{k}(n-1, \ell-1)+(\ell-1) \sum_{j=\ell}^{n-1} \sum_{r=0}^{k-2} p^{k-2-r}\left(\begin{array}{c}
j-\ell \\
r
\end{array}\right)\left(\begin{array}{c}
k-1 \\
r+1
\end{array}\right) a_{k}(n-1, j) \\
& +\sum_{j=\ell}^{n-1} \sum_{i=0}^{k-2} \sum_{r=0}^{k-2-i} p^{k-2-r} q^{i+1}\left(\begin{array}{c}
j-\ell \\
r
\end{array}\right)\left(\begin{array}{c}
k-2-i \\
r
\end{array}\right) a_{k}(n-1, j),
\end{aligned}
$$

where $a_{k}(n, 0)=\delta_{n, 0}$.

Proof. Suppose $\lambda \in \mathcal{T}_{n, \ell}^{(k)}$ where $1 \leq \ell \leq n$. Consider forming $\lambda$ from a precursor $\lambda^{\prime} \in \mathcal{T}_{n-1}^{(k)}$ (on $[2, n]$ ) by inserting $k$ copies of 1 into $\lambda^{\prime}$ at various places. Note that the $1^{\prime}$ 's must be inserted between units of $\lambda^{\prime}$ or at the very beginning or end of $\lambda^{\prime}$, lest there be an occurrence of 212. First consider the case in which the $k$ 1's are inserted into $\lambda^{\prime}$ as a single run (which is seen to increase the number of units by one). Then there are $p^{k-1}(\ell-1) a_{k}(n-1, \ell-1)$ possibilities if the run of 1 's does not occur at the beginning of $\lambda$ and $p^{k-1} q^{k} a_{k}(n-1, \ell-1)$ possibilities if it does. Combining the two cases then gives the first term on the right side of (1) if $\ell \geq 2$. If $\ell=1$, then one gets a contribution of $p^{n-1} q^{n} \delta_{n, 1}$, which coincides with the first term in this case since $a_{k}(n, 0)=\delta_{n, 0}$.

Now assume that $\lambda$ contains more than one run of 1's and does not start with 1 . Let $\lambda^{\prime}$ contain $j$ units. Then $j \geq \ell$ since insertion of the 1's in this case either preserves or reduces the number of units. Suppose that the leftmost 1 is inserted immediately prior to the $s$-th unit of $\lambda^{\prime}$, where $2 \leq s \leq \ell$. Then the rightmost 1 must directly follow the $(s+j-\ell)$-th unit of $\lambda^{\prime}$, which ensures that $\lambda$ will contain $(s-1)+(j-(s+j-\ell))+1=\ell$ units, as required. Suppose further that 1's are to occur in exactly $r$ of the $j-\ell$ possible positions between the $i$-th and the $(i+1)$-st units of $\lambda^{\prime}$ for $s \leq i<s+j-\ell$. After inserting a 1 in each of these $r$ positions (as well as before the $s$-th unit and after the $(s+j-\ell$ )-th), there are $k-2-r$ 1's left to be distributed, each of which produces a level when inserted into its respective position, which accounts for the factor of $p^{k-2-r}$. Finally, there are $\left(\begin{array}{c}k-2-r+(r+1) \\ r+1\end{array}\right)=\left(\begin{array}{l}k-1 \\ r+1\end{array}\right)$ ways in which to insert these $k-2-r$ 1's into $r+2$ positions between units of $\lambda^{\prime}$. Considering all possible $\ell \leq j \leq n-1$ and $0 \leq r \leq k-2$, and noting that each choice of $s$ yields the same number of possibilities, then gives the second part of (1).

Now assume that $\lambda$ contains more than one run of 1's and starts with 1 . In this case, we must also keep track of the number of 1's inserted at the beginning of $\lambda^{\prime}$. Let this number be denoted by $i+1$, where $0 \leq i \leq k-2$, which accounts for the factor of $q^{i+1}$. Let $j$ and $r$ be as before. Then there 
are $\left(\begin{array}{c}k-2-i \\ r\end{array}\right)$ possible ways in which to insert the additional 1's into $r+1$ positions other than at the beginning. Once $i$ is fixed, then reasoning as before implies that there are

$$
q^{i+1} \sum_{j=\ell}^{n-1} \sum_{r=0}^{k-2-i} p^{k-2-r}\left(\begin{array}{c}
j-\ell \\
r
\end{array}\right)\left(\begin{array}{c}
k-2-i \\
r
\end{array}\right) a_{k}(n-1, j)
$$

possible $\lambda$. Considering all $i$ then gives the third and final part of (1), which completes the proof.

Let $a_{k}(n, \ell)=a_{k}(n, \ell ; 1, q)$. Taking $p=1$ in (1), and applying twice Vandermonde's identity (see, e.g., $[4$, Formula 5.23]), gives

$$
\begin{aligned}
a_{k}(n, \ell)= & \left(\ell-1+q^{k}\right) a_{k}(n-1, \ell-1)+(\ell-1) \sum_{j=\ell}^{n-1}\left(\begin{array}{c}
j-\ell+k-1 \\
k-2
\end{array}\right) a_{k}(n-1, j) \\
& +\sum_{j=\ell}^{n-1} \sum_{i=0}^{k-2} q^{i+1}\left(\begin{array}{c}
j-\ell+k-i-2 \\
k-i-2
\end{array}\right) a_{k}(n-1, j) .
\end{aligned}
$$

Let $a(n, \ell)=a_{2}(n, \ell)$. Taking $k=2$ in (2) yields

$$
a(n, \ell)=\left(\ell-1+q^{2}\right) a(n-1, \ell-1)+(\ell-1+q) \sum_{j=\ell}^{n-1} a(n-1, j), \quad n \geq \ell \geq 1 .
$$

In the next section, we study further properties of $a(n, \ell)$. Note that $a(n)=\sum_{\ell=1}^{n} a(n, \ell)$ gives the distribution of the statistic on $\mathcal{T}_{n}=\mathcal{T}_{n}^{(2)}$ which records the number of wlrm.

In the remainder of this section, we develop an alternative recursive approach to finding $a(n)$. To do so, first let $\mathcal{D}_{n, i, m}$ denote the set of sequences $s=s_{1} s_{2} \cdots s_{n-i+1}$ such that $s_{j} \in[2 n-2 j+1]$ for $1 \leq j \leq n-i+1$ with $m=\min \left(s_{1} s_{2} \cdots s_{n-i+1}\right)$, where $n \geq 1,1 \leq i \leq n$ and $1 \leq m \leq 2 i-1$. Let $d(n, i, m)=\left|\mathcal{D}_{n, i, m}\right|$. We define a $q$-generalization of $d(n, i, m)$ as follows. By a substantial left-right minimum within a sequence $t=t_{1} t_{2} \cdots$, we mean an index $i$ such that $t_{i}<t_{j}-1$ for all $1 \leq j<i$, with this vacuously holding if $i=1$. By a marginal left-right minimum, we mean an index such that $t_{i}<t_{j}$ for all $1 \leq j<i$ with $t_{i}=t_{j}-1$ for at least one such $j$. We will use similar terminology when referring to right-left minima. Let $\sigma_{1}(\lambda)$ and $\sigma_{2}(\lambda)$ denote the number of substantial and marginal left-right minima, respectively, of $\lambda \in \mathcal{D}_{n, i, m}$. Define the distribution $d_{q}(n, i, m)$ on $\mathcal{D}_{n, i, m}$ by

$$
d_{q}(n, i, m)=\sum_{\lambda \in \mathcal{D}_{n, i, m}} q^{2 \sigma_{1}(\lambda)+\sigma_{2}(\lambda)} .
$$

Then the polynomials $d_{q}(n, i, m)$ are determined recursively as follows.

Proposition 2.2 If $1 \leq i<n$ and $1 \leq m \leq 2 i-1$, then

$$
d_{q}(n, i, m)=(2 i-m) d_{q}(n, i+1, m)+q a_{q}(n, i+1, m+1)+q^{2} \sum_{j=m+2}^{2 i+1} d_{q}(n, i+1, j),
$$

with $d_{q}(n, n, m)=q^{2}$ for all $n \geq 1$ and $1 \leq m \leq 2 n-1$. 
Proof. Since the first letter of any word is regarded as a substantial but not a marginal left-right minimum, the initial condition when $i=n$ follows from the definitions. Let $\delta \in \mathcal{D}_{n, i, m}$ where $1 \leq i<n$. Then $\delta$ may be obtained from a precursor $\delta^{\prime}=d_{1} d_{2} \cdots d_{n-i} \in \mathcal{D}_{n, i+1, j}$ for some $j$ by appending a final letter $x$. If $x<j-1$, then $x$ contributes a substantial left-right minimum and $x=m<j-1$, i.e., $m+2 \leq j \leq 2 i+1$. Considering all possible $j$ yields $q^{2} \sum_{j=m+2}^{2 i+1} d_{q}(n, i+1, j)$ possibilities for $\delta$ in this case. If $x<d_{r}$ for all $r$ with $x=d_{r}-1$ for at least one $r \in[n-i]$, then $x=m$ is a marginal left-right minimum and $j=m+1$, which yields $q d_{q}(n, i+1, m+1)$ possibilities. Finally, if $x \geq j$, then $j=m$ and there are $(2 i-1)-(m-1)=2 i-m$ choices for $x$, which gives the remaining term on the right side of (4) and completes the proof.

The special case of $d_{q}(n, i, m)$ when $i=m=1$ corresponds to the distribution of wlrm on $\mathcal{T}_{n}$.

Proposition 2.3 If $n \geq 1$, then $d_{q}(n, 1,1)=\sum_{\ell=1}^{n} a(n, \ell)$.

Proof. Let $s \in \mathcal{D}_{n, 1,1}$ and $s^{\prime}=s_{1}^{\prime} s_{2}^{\prime} \cdots s_{n}^{\prime}$ denote the reversal of $s$; note that $s_{i}^{\prime} \in[2 i-1]$ for $1 \leq i \leq n$. We form a member $w \in \mathcal{T}_{n}$ using $s^{\prime}$ by first writing two copies of 1 . For each successive $i \in[2, n]$, starting with $i=2$, insert two copies of $i$ into the current member of $\mathcal{T}_{i-1}$ by inserting two $i$ 's in one of $2 i-1$ possible positions according to the value of $s_{i}^{\prime}$. Note that these are the only possibilities regarding the placement of the $i$ 's since they must be adjacent, for otherwise there is an occurrence of the pattern 212. Note that if the letter $y$ in the $\ell$-th position of $s^{\prime}$ corresponds to a substantial right-left minimum, which means all letters beyond the $\ell$-th position are greater than $y+1$, then both copies of the letter $\ell$ in $w$ are wlrm since all copies of letters in $[\ell+1, n]$ must occur to the right of the second $\ell$. If the letter $y$ in $s^{\prime}$ corresponds to a marginal right-left minimum, then no letter greater than $\ell$ appears to the left of $\ell$ in $w$ with at least one letter in $[\ell+1, n]$ occurring between the two copies of $\ell$, which implies only the first $\ell$ is a wlrm. Finally, if the letter $y$ is neither a substantial nor a marginal right-left minimum, then some letter greater than $\ell$ appears to the left of $\ell$ in $w$, which implies neither copy of $\ell$ is a wlrm. Thus, since the mapping $s \mapsto w$ is seen to be a bijection, it follows that the distribution of $2 \sigma_{1}+\sigma_{2}$ on $\mathcal{D}_{n, 1,1}$ is the same as the wlrm statistic on $\mathcal{T}_{n}$, which implies the desired result.

\section{Generating function for distribution on Stirling permutations}

In this section, we find an explicit formula for the generating function of $a_{k}(n, \ell ; 1, q)$ and $a_{k}(n, \ell ; p, q)$ in the case $k=2$. Recall that $a(n, \ell)$ denotes the quantity $a_{2}(n, \ell ; 1, q)$. Define $A(x ; \ell)=\sum_{n \geq \ell} a(n, \ell) \frac{x^{n}}{n !}$. Then recurrence (3) above can be rewritten in terms of generating functions as

$$
\frac{d}{d x} A(x ; \ell)=\left(\ell-1+q^{2}\right) A(x ; \ell-1)+(\ell-1+q) \sum_{j \geq \ell} A(x ; j), \quad \ell \geq 1,
$$

with $A(x ; 0)=1$. Before considering $A(x ; \ell)$ for all $q$, note that when $q=1$ in $(5)$, we have

$$
\left.\frac{d}{d x} A(x ; \ell)\right|_{q=1}=\left.\ell \sum_{j \geq \ell-1} A(x ; j)\right|_{q=1}, \quad \ell \geq 1,
$$

with $\left.A(x ; 0)\right|_{q=1}=1$. Using (6), one may verify

$$
\left.A(x ; \ell)\right|_{q=1}=(1-\sqrt{1-2 x})^{\ell}, \quad \ell \geq 0 .
$$


Summing this over $\ell$ implies $\left.\sum_{\ell \geq 0} A(x ; \ell)\right|_{q=1}=\frac{1}{\sqrt{1-2 x}}$, which coincides with the well-known formula for the exponential generating function for the number of perfect matchings of $[2 n]$.

Let $\mathbf{v}(x)$ be the column vector $(A(x ; 0), A(x ; 1), \ldots)^{T}$, where $q$ now is arbitrary. Define $\mathbf{A}$ to be the infinite matrix

$$
\mathbf{A}=\left(\begin{array}{ccccc}
0 & 0 & 0 & 0 & \cdots \\
q^{2} & q & q & q & \cdots \\
0 & 1+q^{2} & 1+q & 1+q & \cdots \\
0 & 0 & 2+q^{2} & 2+q & \cdots \\
\vdots & \vdots & \ddots & \ddots &
\end{array}\right)
$$

Note that $\frac{d}{d x} \mathbf{v}(x)=\mathbf{A v}(x)$.

Proposition 3.1 The generating functions

$$
A(x, y)=\sum_{n \geq 0} \sum_{\ell=0}^{n} a(n, \ell) x^{n} y^{\ell} \text { and } E(x, y)=\sum_{n \geq 0} \sum_{\ell=0}^{n} a(n, \ell) \frac{x^{n} y^{\ell}}{n !}
$$

are given by

$$
A(x, y)=\left(1, y, y^{2}, \ldots\right)(1-\mathbf{A} x)^{-1} e_{1}^{T}
$$

and

$$
E(x, y)=\left(1, y, y^{2}, \ldots\right) e^{\mathbf{A} x} e_{1}^{T},
$$

where $e_{1}=(1,0,0, \ldots)$.

Proof. From the fact $\frac{d}{d x} \mathbf{v}(x)=\mathbf{A v}(x)$, we have $\left.\frac{d^{m}}{d x^{m}} \mathbf{v}(x)\right|_{x=0}=\mathbf{A}^{m} \mathbf{v}(0)$. Note that $\mathbf{v}(0)=e_{1}^{T}=$ $(1,0,0, \ldots)^{T}$, which implies

$$
A(x, y)=\left(1, y, y^{2}, \ldots\right)\left(\sum_{m \geq 0} \mathbf{A}^{m} x^{m}\right) e_{1}^{T}=\left(1, y, y^{2}, \ldots\right)(1-\mathbf{A} x)^{-1} e_{1}^{T}
$$

and

$$
E(x, y)=\left(1, y, y^{2}, \ldots\right)\left(\sum_{m \geq 0} \mathbf{A}^{m} \frac{x^{m}}{m !}\right) e_{1}^{T}=\left(1, y, y^{2}, \ldots\right) e^{\mathbf{A} x} e_{1}^{T},
$$

as required.

Define

$$
\mathbf{A}^{\prime}=1-x \mathbf{A}=\left(\begin{array}{ccccc}
1 & 0 & 0 & 0 & \cdots \\
-x q^{2} & 1-x q & -x q & -x q & \cdots \\
0 & -x\left(1+q^{2}\right) & 1-x(1+q) & -x(1+q) & \cdots \\
0 & 0 & -x\left(2+q^{2}\right) & 1-x(2+q) & \cdots \\
\vdots & \vdots & \ddots & \ddots &
\end{array}\right)
$$

and we seek to determine the inverse matrix of $\mathbf{A}^{\prime}$. In order to do so, first let $\mathbf{A}^{\prime}=\mathbf{L} \mathbf{U}$ be the $L U$-decomposition of the matrix $\mathbf{A}^{\prime}$. Let $\mathbf{A}_{n}^{\prime}$ be the submatrix of $\mathbf{A}^{\prime}$ on the first $n$ rows and $n$ columns and denote its determinant by $d_{n}$; that is, $d_{n}=\operatorname{det}\left(\mathbf{A}_{n}^{\prime}\right)$ if $n \geq 1$, with $d_{0}=1$. 
LEMma 3.2 We have

$$
d_{n}=(1+x q(q-1)) d_{n-1}-x\left(n-2+q^{2}\right) d_{n-2}, \quad n \geq 2,
$$

with $d_{0}=d_{1}=1$. Moreover,

$$
\sum_{n \geq 1} \frac{d_{n}}{\prod_{i=1}^{n-1}\left(i+q^{2}\right)} y^{n}=q^{2} y^{1-q^{2}} e^{y\left(q^{2} x-q x-x y / 2+1\right)} \int_{0}^{y}(1-t x) t^{q^{2}-1} e^{-t\left(q^{2} x-t x / 2-q x+1\right)} d t .
$$

Proof. Using repeated cofactor expansion along the last row to compute the determinant, we have

$$
d_{n}=d_{n-1}-\sum_{j=1}^{n-1} x^{n-j} d_{j}(j-1+q) \prod_{i=j}^{n-2}\left(i+q^{2}\right), \quad n \geq 1,
$$

with $d_{0}=1$. If $n \geq 2$, then

$$
\begin{aligned}
d_{n}-d_{n-1} & =-x d_{n-1}(n-2+q)-x\left(n-2+q^{2}\right) \sum_{j=1}^{n-2} x^{n-1-j} d_{j}(j-1+q) \prod_{i=j}^{n-3}\left(i+q^{2}\right) \\
& =-x d_{n-1}(n-2+q)+x\left(n-2+q^{2}\right)\left(d_{n-1}-d_{n-2}\right),
\end{aligned}
$$

which gives (7). Now define $e_{n}$ by $d_{n}=e_{n} \prod_{i=1}^{n-1}\left(i+q^{2}\right)$. Then (8) can be rewritten as

$$
e_{n}\left(n-1+q^{2}\right)=e_{n-1}-\sum_{j=1}^{n-1} x^{n-j} e_{j}(j-1+q), \quad n \geq 2
$$

with $e_{1}=1$. If $E(y)=\sum_{n \geq 1} e_{n} y^{n}$, then we get

$$
y \frac{d}{d y}(E(y)-y)+\left(q^{2}-1\right)(E(y)-y)=y E(y)-\frac{(q-1) x y}{1-x y} E(y)-\frac{x y^{2}}{1-x y} \frac{d}{d y} E(y),
$$

which is equivalent to

$$
y \frac{d}{d y} E(y)+\left(\left(q^{2}-q-y\right)(1-x y)+q-1\right) E(y)=q^{2} y(1-x y) .
$$

The solution of this differential equation with initial condition $E(0)=0$ is given by

$$
E(y)=q^{2} y^{1-q^{2}} e^{y\left(q^{2} x-q x-x y / 2+1\right)} \int_{0}^{y}(1-t x) t^{q^{2}-1} e^{-t\left(q^{2} x-t x / 2-q x+1\right)} d t
$$

as required.

Remark. Note that when $q=1$, we have $\left.E(y)\right|_{q=1}=e^{y(1-x y / 2)} \int_{0}^{y}(1-t x) e^{-t(1-t x / 2)} d t$, which leads to $\left.E(y)\right|_{q=1}=e^{y(1-x y / 2)}-1$. Hence,

$$
\sum_{n \geq 0} \frac{\left.d_{n}\right|_{q=1}}{n !} y^{n}=e^{y-x y^{2} / 2} .
$$

The matrix $\mathbf{A}^{\prime}$ has the following $L U$-decomposition. 
Lemma 3.3 We have $\mathbf{A}^{\prime}=\mathbf{L} \mathbf{U}$, where $\mathbf{L}=\left(\ell_{i j}\right)$ and $\mathbf{U}=\left(u_{i j}\right)$ are given by

$$
\ell_{i j}=\left\{\begin{array}{ll}
1, & j=i \geq 1, \\
\frac{-x\left(i-2+q^{2}\right) d_{i-2}}{d_{i-1}}, & j=i-1 \geq 1, \\
0, & \text { otherwise, }
\end{array} \quad \text { and } u_{i j}= \begin{cases}\frac{d_{i}}{d_{i-1}}, & j=i \geq 1, \\
\frac{d_{i}}{d_{i-1}}-1, & 2 \leq i+1 \leq j, \\
0, & \text { otherwise }\end{cases}\right.
$$

Proof. Let $a_{i j}^{\prime}$ denote the $(i, j)$ entry of $\mathbf{A}^{\prime}$. We compute $\sum_{r \geq 1} \ell_{i r} u_{r j}$ in several cases and compare with $a_{i j}^{\prime}$. If $j \leq i-2$, then both quantities are zero, so assume $j \geq i-1$. If $j=i-1$, then

$$
\sum_{r \geq 1} \ell_{i r} u_{r j}=\ell_{i i} u_{i, i-1}+\ell_{i, i-1} u_{i-1, i-1}=0-\frac{x\left(i-2+q^{2}\right) d_{i-2}}{d_{i-1}} \cdot \frac{d_{i-1}}{d_{i-2}}=-x\left(i-2+q^{2}\right)=a_{i j}^{\prime} .
$$

If $i=j=1$, then both entries are clearly 1 , so assume $i=j \geq 2$. Then we have

$$
\begin{aligned}
\ell_{i i} u_{i i}+\ell_{i, i-1} u_{i-1, i} & =\frac{d_{i}}{d_{i-1}}+\left(\frac{-x\left(i-2+q^{2}\right) d_{i-2}}{d_{i-1}}\right)\left(\frac{d_{i-1}}{d_{i-2}}-1\right) \\
& =\frac{d_{i}+x\left(i-2+q^{2}\right) d_{i-2}}{d_{i-1}}-x\left(i-2+q^{2}\right)
\end{aligned}
$$

which equals $a_{i i}^{\prime}=1-x(i-2+q)$, upon using (7). If $i=1$ and $j \geq 2$, then both entries are again zero since $d_{0}=d_{1}=1$. Finally, if $j \geq i+1$ with $i \geq 2$, then the $(i, j)$ entry of the product $\mathbf{L} \mathbf{U}$ is the same quantity as in the case $i=j \geq 2$ above except that there is an extra minus 1 term. Thus, it works out to $-x(i-2+q)$, which is again the same as $a_{i, j}^{\prime}$. Combining the previous cases now implies the result.

Thus we have $\mathbf{A}^{\prime-\mathbf{1}}=\mathbf{U}^{-\mathbf{1}} \mathbf{L}^{-\mathbf{1}}$, where the inverse matrices $\mathbf{L}^{-1}=\left(\tilde{\ell}_{i j}\right)$ and $\mathbf{U}^{-1}=\left(\tilde{u}_{i j}\right)$ are given explicitly by

$$
\tilde{\ell}_{i j}=\left\{\begin{array}{ll}
1, & j=i, \\
\frac{d_{j-1} x^{i-j} \prod_{s=j+1}^{i}\left(s-2+q^{2}\right)}{d_{i-1}}, & \begin{array}{l}
1 \leq j \leq i-1, \\
0,
\end{array} \text { otherwise }
\end{array} \quad \text { and } \tilde{u}_{i j}= \begin{cases}\frac{d_{i-1}}{d_{i}}, & i=j, \\
-\frac{d_{i}-d_{i-1}}{d_{j}}, & 2 \leq i+1 \leq j, \\
0, & \text { otherwise }\end{cases}\right.
$$

which may be verified by a direct computation using the formulas from Lemma 3.3.

The $(i, 1)$ term for $i \geq 2$ in the product of matrices $\mathbf{U}^{-1} \mathbf{L}^{-1}$ is given by

$$
\begin{aligned}
\left(\mathbf{U}^{-1} \mathbf{L}^{-1}\right)_{i 1} & =\sum_{k \geq 1} \tilde{u}_{i k} \tilde{\ell}_{k 1}=\frac{x^{i-1} \prod_{s=0}^{i-2}\left(s+q^{2}\right)}{d_{i}}+\sum_{k \geq i+1} \tilde{u}_{i k} \tilde{\ell}_{k 1} \\
& =\frac{x^{i-1} \prod_{s=0}^{i-2}\left(s+q^{2}\right)}{d_{i}}-\sum_{k \geq i+1} \frac{d_{i}-d_{i-1}}{d_{k}} \frac{x^{k-1} \prod_{s=2}^{k}\left(s-2+q^{2}\right)}{d_{k-1}} \\
& =\frac{x^{i-1} \prod_{s=0}^{i-2}\left(s+q^{2}\right)}{d_{i}}\left(1-d_{i}\left(d_{i}-d_{i-1}\right) \sum_{k \geq i+1} \frac{\prod_{s=i-1}^{k-2}\left(s+q^{2}\right)}{d_{k-1} d_{k}} x^{k-i}\right),
\end{aligned}
$$

which is seen to hold also for $i=1$ since $d_{0}=d_{1}=1$. By Proposition 3.1 and Lemma 3.2, we have the following result. 
THEOREM 3.4 The generating function $A(x, y)=\sum_{n \geq 0} \sum_{\ell=0}^{n} a(n, \ell) x^{n} y^{\ell}$ is given by

$$
\begin{aligned}
A(x, y) & =\left(1, y, y^{2}, \ldots\right) \mathbf{U}^{-1} \mathbf{L}^{-1}(1,0,0, \ldots)^{T} \\
& =\sum_{i \geq 1}\left(\frac{(x y)^{i-1} \prod_{s=0}^{i-2}\left(s+q^{2}\right)}{d_{i}}\left(1-d_{i}\left(d_{i}-d_{i-1}\right) \sum_{k \geq i+1} \frac{\prod_{s=i-1}^{k-2}\left(s+q^{2}\right)}{d_{k-1} d_{k}} x^{k-i}\right)\right),
\end{aligned}
$$

where $d_{n}$ is determined by (7).

Remark. Let $d_{n}=d_{n}(x, q)$, where $d_{n}$ is as above, and let $d_{n}(x)=d_{n}(x, 1)$. Note that Theorem 3.4 when $q=1$ gives

$$
\left.A(x, y)\right|_{q=1}=\sum_{i \geq 1}\left(\frac{(x y)^{i-1}(i-1) !}{d_{i}(x)}\left(1-\frac{d_{i}(x)\left(d_{i}(x)-d_{i-1}(x)\right)}{(i-1) !} \sum_{k \geq i+1} \frac{(k-1) !}{d_{k-1}(x) d_{k}(x)} x^{k-i}\right)\right) .
$$

It would be interesting to find explicit formulas for $\sum_{i \geq 1} \frac{(i-1) ! y^{i-1}}{d_{i}(x)}$ and $\sum_{k \geq i+1} \frac{(k-1) ! x^{k-1}}{d_{k}(x) d_{k-1}(x)}$.

The preceding can be generalized as follows. Let $a(n, \ell ; p)=a_{2}(n, \ell ; p, q)$; note that $a(n, \ell ; 1)=$ $a(n, \ell)$. Taking $k=2$ in (1) gives the recurrence

$$
a(n, \ell ; p)=p\left(\ell-1+q^{2}\right) a(n-1, \ell-1 ; p)+(\ell-1+q) \sum_{j=\ell}^{n-1} a(n-1, j ; p), \quad n \geq \ell \geq 1,
$$

which reduces to $(3)$ when $p=1$. Similarly, let us define the generating function $A(x ; \ell)=A_{p}(x ; \ell)$ by $A(x ; \ell)=\sum_{n \geq \ell} a(n, \ell ; p) \frac{x^{n}}{n !}$. Then $(9)$ can be written as

$$
\frac{d}{d x} A(x ; \ell)=p\left(\ell-1+q^{2}\right) A(x ; \ell-1)+(\ell-1+q) \sum_{j \geq \ell} A(x ; j), \quad \ell \geq 1 .
$$

Note that (10) when $q=1$ gives

$$
\frac{d}{d x} A(x ; \ell)=p \ell A(x ; \ell-1)+\ell \sum_{j \geq \ell} A(x ; j),
$$

with $A(x ; 0)=1$. This leads to the formula

$$
\left.A(x ; \ell)\right|_{q=1}=\left(\frac{W\left(-e^{(p-1)^{2} x-1}\right)+p}{p-1}\right)^{\ell},
$$

where $W(t)$ is the Lambert's function defined by $W(x)=e^{-W(x)}$.

One may also find $A(x ; \ell)$ for arbitrary $p$ and $q$ by extending the prior arguments which we now describe. Let $\mathbf{v}(x)$ be the column vector $(A(x ; 0), A(x ; 1), \ldots)^{T}$ and define $\mathbf{A}$ to be the infinite matrix

$$
\mathbf{A}=\left(\begin{array}{ccccc}
0 & 0 & 0 & 0 & \cdots \\
p q^{2} & q & q & q & \cdots \\
0 & p\left(1+q^{2}\right) & 1+q & 1+q & \cdots \\
0 & 0 & p\left(2+q^{2}\right) & 2+q & \cdots \\
\vdots & \vdots & \ddots & \ddots &
\end{array}\right)
$$


Note that $\frac{d}{d x} \mathbf{v}(x)=\mathbf{A v}(x)$. By using techniques similar to the proof of Proposition 3.1 above, one can show that the generating functions

$$
A(x, y)=\sum_{n \geq 0} \sum_{\ell=0}^{n} a(n, \ell ; p) x^{n} y^{\ell} \text { and } E(x, y)=\sum_{n \geq 0} \sum_{\ell=0}^{n} a(n, \ell ; p) \frac{x^{n} y^{\ell}}{n !}
$$

are given by

$$
A(x, y)=\left(1, y, y^{2}, \ldots\right)(1-\mathbf{A} x)^{-1} e_{1}^{T}
$$

and

$$
E(x)=\left(1, y, y^{2}, \ldots\right) e^{\mathbf{A} x} e_{1}^{T},
$$

where $e_{1}=(1,0,0, \ldots)$.

Let

$$
\mathbf{A}^{\prime}=1-x \mathbf{A}=\left(\begin{array}{ccccc}
1 & 0 & 0 & 0 & \ldots \\
-x p q^{2} & 1-x q & -x q & -x q & \ldots \\
0 & -x p\left(1+q^{2}\right) & 1-x(1+q) & -x(1+q) & \ldots \\
0 & 0 & -x p\left(2+q^{2}\right) & 1-x(2+q) & \ldots \\
\vdots & \vdots & \ddots & \ddots &
\end{array}\right)
$$

and let $\mathbf{A}_{n}^{\prime}$ and $d_{n}$ be defined in a comparable manner as before.

LEMMA 3.5 We have

$$
d_{n}=(1+x(p-1)(n-2)+x q(p q-1)) d_{n-1}-x p\left(n-2+q^{2}\right) d_{n-2}, \quad n \geq 2,
$$

with $d_{0}=d_{1}=1$. Moreover,

$$
\begin{aligned}
& \sum_{n \geq 1} \frac{d_{n}}{p^{n-1} \prod_{i=1}^{n-1}\left(i+q^{2}\right)} y^{n} \\
& =p q^{2} y^{1-q^{2}}(x y(1-p)+p)^{\frac{q^{2}-q}{1-p}+\frac{1}{x(1-p)^{2}}} e^{-\frac{y}{1-p}} \int_{0}^{y} t^{q^{2}-1}(1-x t)(x t(1-p)+p)^{-1-\frac{q(q-1)}{1-p}-\frac{1}{x(1-p)^{2}}} e^{\frac{t}{1-p}} d t .
\end{aligned}
$$

Proof. Expanding the determinant repeatedly along the last row implies

$$
d_{n}=d_{n-1}-\sum_{j=1}^{n-1} p^{n-1-j} x^{n-j} d_{j}(j-1+q) \prod_{i=j}^{n-2}\left(i+q^{2}\right),
$$

from which (11) follows. Now let $d_{n}=p^{n-1} e_{n} \prod_{i=1}^{n-1}\left(i+q^{2}\right)$. Then

$$
p e_{n}\left(n-1+q^{2}\right)=e_{n-1}-\sum_{j=1}^{n-1} x^{n-j} e_{j}(j-1+q), \quad n \geq 2,
$$

with $e_{1}=1$. If $E(y)=\sum_{n \geq 1} e_{n} y^{n}$, then

$$
p y \frac{d}{d y}(E(y)-y)+p\left(q^{2}-1\right)(E(y)-y)=y E(y)-\frac{(q-1) x y}{1-x y} E(y)-\frac{x y^{2}}{1-x y} \frac{d}{d y} E(y) .
$$


The solution of this differential equation with $E(0)=0$ and $\left.\frac{d}{d y} E(y)\right|_{y=0}=p q$ is given by

$$
\begin{aligned}
E(y)=p q^{2} y^{1-q^{2}}(x y(1-p) & +p)^{\frac{q^{2}-q}{1-p}+\frac{1}{x(1-p)^{2}} e^{-\frac{y}{1-p}}} \\
& \times \int_{0}^{y} t^{q^{2}-1}(1-x t)(x t(1-p)+p)^{-1-\frac{q(q-1)}{1-p}-\frac{1}{x(1-p)^{2}} e^{\frac{t}{1-p}}} d t
\end{aligned}
$$

as required.

Proceeding similarly as in the proof of Theorem 3.4 above, we obtain the following result.

THEOREM 3.6 The generating function $A(x, y)=\sum_{n \geq 0} \sum_{\ell=0}^{n} a(n, \ell ; p) x^{n} y^{\ell}$ is given by

$$
A(x, y)=\sum_{i \geq 1}\left(\frac{(p x y)^{i-1} \prod_{s=0}^{i-2}\left(s+q^{2}\right)}{d_{i}}\left(1-p d_{i}\left(d_{i}-d_{i-1}\right) \sum_{k \geq i+1} \frac{\prod_{s=i-1}^{k-2}\left(s+q^{2}\right)}{d_{k-1} d_{k}}(p x)^{k-i}\right)\right),
$$

where $d_{n}$ is determined by (11).

\section{References}

[1] K. Archer, A. Gregory, B. Pennington and S. Slayden, Pattern restricted quasi-Stirling permutations, Australas. J. Combin., 74 (2019) 389-407.

[2] G.-H. Duh, Y.-C. R. Lin, S.-M. Ma And Y.-N. Yeh, Some statistics on Stirling permutations and Stirling derangements, Discrete Math., 341 (2018) 2478-2484.

[3] I. Gessel and R. P. Stanley, Stirling polynomials, J. Combin. Theory Ser. A, 24 (1978) $25-33$.

[4] R. L. Graham, D. E. Knuth and O. Patashnik, Concrete Mathematics: A Foundation for Computer Science, Second Edition, Addison-Wesley, Boston, 1994.

[5] J. Haglund And M. Visontai, Stable multivariate Eulerian polynomials and generalized Stirling permutations, European J. Combin., 33 (2012) 477-487.

[6] S.-M. Ma, J. Ma AND Y.-N. YeH, The ascent-plateau statistics on Stirling permutations, Electron. J. Combin., 26 (2019) \#P2.5.

[7] S.-M. Ma AND T. Mansour, The $1 / k$-Eulerian polynomials and $k$-Stirling permutations, Discrete Math., 338 (2015) 1468-1472.

[8] S.-M. Ma And T. Mansour, Pattern restricted Stirling $k$-ary words, the plateau statistic and the kernel method, Discrete Appl. Math., 206 (2016) 100-108.

[9] J. B. Remmel and A. T. Wilson, Block patterns in Stirling permutations, J. Comb., 6 (2015) 179-204.

[10] N. J. A. Sloane, The On-Line Encyclopedia of Integer Sequences, at http://oeis.org. 